IANA Allocation Guidelines For Values In

the Internet Protocol and Related Headers

Status of this Memo

This document specifies an Internet Best Current Practices for the Internet Community, and requests discussion and suggestions for improvements. Distribution of this memo is unlimited.

Copyright Notice

Copyright (C) The Internet Society (2000). All Rights Reserved.

Abstract

This memo provides guidance for the IANA to use in assigning parameters for fields in the IPv4, IPv6, ICMP, UDP and TCP protocol headers.

1. Introduction

For many years the Internet Assigned Numbers Authority (IANA) (www.iana.org) has allocated parameter values for fields in protocols which have been created or are maintained by the Internet Engineering Task Force (IETF). Starting a few years ago the IETF began to provide the IANA with guidance for the assignment of parameters for fields in newly developed protocols. Unfortunately this type of guidance was not consistently provided for the fields in protocols developed before 1998. This memo attempts to codify existing IANA practice used in the assignment of parameters in the specific case of some of these protocols. It is expected that additional memos will be developed in the future to codify existing practice in other cases.

This memo addresses the fields within the IPv4, IPv6, ICMP, UDP and TCP protocol headers for which the IANA assigns values.

The terms "Specification Required", "Expert Review", "IESG Approval", "IETF Consensus", and "Standards Action", are used in this memo to refer to the processes described in [CONS]. 


\section{Temporary Assignments}

From time to time temporary assignments are made in the values for fields in these headers for use in experiments. IESG Approval is required for any such temporary assignments.

3. Version field in the IP header.

The first field in the IP header of all current versions of IP is the Version field. New values in the Version field define new versions of the IP protocol and are allocated only after an IETF Standards Action. It should be noted that some of the Version number bits are used by TCP/IP header compression schemes. Specifically, the hi-order bit of the Version field is also used by TCP/IP header compression [HC], while the three hi-order bits are used by IP Header Compression [IPHC] .

4. IANA Considerations for fields in the IPv4 header

The IPv4 header [V4] contains the following fields that carry values assigned by the IANA: Version, Type of Service, Protocol, Source Address, Destination Address, and Option Type.

4.1 IPv4 IP Version field

The IPv4 Version field is always 4.

4.2 IPv4 Type of Service field

The Type of Service field described in [V4] has been superseded[DIFF] by the 6-bit Differentiated Services (DS) field and a 2-bit field which is currently reserved. The IANA allocates values in the DS field following the IANA Considerations section in [DIFF]. [ECN] describes an experimental use of the 2-bit "currently unused" field. Other experimental uses of this field may be assigned after IESG Approval processes. Permanent values in this field are allocated following a Standards Action process.

\subsection{IPv4 Protocol field}

IANA allocates values from the IPv4 Protocol name space following an Expert Review, IESG Approval or Standards Action process. The Expert Review process should only be used in those special cases where nondisclosure information is involved. In these cases the expert(s) should be designated by the IESG. 


\subsection{IPv4 Source and Destination addresses}

The IPv4 source and destination addresses use the same namespace but do not necessarily use the same values. Values in these fields fall into a number of ranges defined in [V4] and [MULT].

\subsubsection{IPv4 Unicast addresses}

The Internet Corporation for Assigned Names and Numbers (ICANN) recently accepted responsibility for the formulation of specific guidelines for the allocation of the values from the IPv4 unicast address space (values 0.0.0.0 through 223.255.255.255) other than values from the ranges $0 / 8$ (which was reserved in [AN80]) and 127/8 (from which the loopback address has been taken) along with other values already assigned by the IETF for special functions or purposes. (For example, the private addresses defined in RFC 1918.) Further assignments in the $0 / 8$ and $127 / 8$ ranges require a standards Action process since current IP implementations may break if this is done.

\subsubsection{IPv4 Multicast addresses}

IPv4 addresses that fall in the range from 224.0 .0 .0 through 239.255.255.255 are known as multicast addresses. The IETF through its normal processes has assigned a number of IPv4 multicast addresses for special purposes. For example, [ADSCP] assigned a number of IPv4 multicast address to correspond to IPv6 scoped multicast addresses. Also, the values in the range from 224.0.0.0 to 224.0.0.255, inclusive, are reserved by the IANA for the use of routing protocols and other low-level topology discovery or maintenance protocols, such as gateway discovery and group membership reporting. (See the IANA web page) New values in this range are assigned following an IESG Approval or Standards Action process. Assignments of individual multicast address follow an Expert Review, IESG Approval or Standards Action process. Until further work is done on multicast protocols, large-scale assignments of IPv4 multicast addresses is not recommended.

From time to time, there are requests for temporary assignment of multicast space for experimental purposes. These will originate in an IESG Approval process and should be for a limited duration such as one year.

\subsubsection{IPv4 Reserved addresses}

IPv4 addresses in the range from 240.0.0.0 through 255.255.255.254 are reserved [AN81, MULT] and compliant IPV4 implementations will discard any packets that make use of them. Addresses in this range 
are not to be assigned unless an IETF Standards Action modifies the IPv4 protocol in such a way as to make these addresses valid. Address 255.255.255.255 is the limited broadcast address.

\subsection{IPv4 Option Type field}

The IANA allocates values from the IPv4 Option Type name space following an IESG Approval, IETF Consensus or Standards Action process.

5. IANA Considerations for fields in the IPv6 header

The IPv6 header [V6] contains the following fields that carry values assigned from IANA-managed name spaces: Version (by definition always 6 in IPv6), Traffic Class, Next Header, Source and Destination Address. In addition, the IPv6 Hop-by-Hop Options and Destination Options extension headers include an Option Type field with values assigned from an IANA-managed name space.

5.1 IPv6 Version field

The IPv6 Version field is always 6.

5.2 IPv6 Traffic Class field

The IPv6 Traffic Class field is described in [DIFF] as a 6- bit Differentiated Services (DS) field and a 2-bit field which is currently reserved. See Section 4.2 for assignment guidelines for these fields.

5.3 IPv6 Next Header field

The IPv6 Next Header field carries values from the same name space as the IPv4 Protocol name space. These values are allocated as discussed in section 4.3 .

\subsection{IPv6 Source and Destination Unicast Addresses}

The IPv6 Source and Destination address fields both use the same values and are described in [V6AD]. The addresses are divided into ranges defined by a variable length Format Prefix (FP) .

\subsubsection{IPv6 Aggregatable Global Unicast Addresses}

The IANA was given responsibility for all IPv6 address space by the IAB in [V6AA]. Recently the IANA agreed to specific guidelines for the assignment of values in the Aggregatable Global Unicast Addresses FP (FP 001) formulated by the Regional Internet Registries. 


\subsubsection{IPv6 Anycast Addresses}

IPv6 anycast addresses are defined in [V6AD]. Anycast addresses are allocated from the unicast address space and anycast addresses are syntactically indistinguishable from unicast addresses. Assignment of IPv6 Anycast subnet addresses follows the process described in [V6AD]. Assignment of other IPv6 Anycast addresses follows the process used for IPv6 Aggregatable Global Unicast Addresses. (section 5.4.1)

\subsubsection{IPv6 Multicast Addresses}

IPv6 multicast addresses are defined in [V6AD]. They are identified by a FP of 0xFF. Assignment guidelines for IPv6 multicast addresses are described in [MASGN].

\subsubsection{IPv6 Unassigned and Reserved IPv6 Format Prefixes}

The responsibility for assigning values in each of the "unassigned" and "reserved" Format Prefixes is delegated by IESG Approval or Standards Action processes since the rules for processing these Format Prefixes in IPv6 implementations have not been defined.

5.5 IPv6 Hop-by-Hop and Destination Option Fields

Values for the IPv6 Hop-by-Hop Options and Destination Options fields are allocated using an IESG Approval, IETF Consensus or Standards Action processes.

\section{6 IPv6 Neighbor Discovery Fields}

The IPv6 Neighbor Discovery header [NDV6] contains the following fields that carry values assigned from IANA- managed name spaces: Type, code and Option Type.

Values for the IPv6 Neighbor Discovery Type, Code, and Option Type fields are allocated using an IESG Approval or Standards Action process.

6. IANA Considerations for fields in the IPv4 ICMP header

The IPV4 ICMP header [ICMP] contains the following fields that carry values assigned from IANA-managed name spaces: Type and Code. Code field values are defined relative to a specific Type value.

Values for the IPv4 ICMP Type fields are allocated using an IESG Approval or Standards Action processes. Code Values for existing IPv4 ICMP Type fields are allocated using IESG Approval or Standards 
Action processes. The policy for assigning Code values for new IPv4 ICMP Types should be defined in the document defining the new Type value.

7. IANA Considerations for fields in the IPv6 ICMP header

The IPv6 ICMP header [ICMPV6] contains the following fields that carry values assigned from IANA-managed name spaces: Type and Code. Code field values are defined relative to a specific Type value.

Values for the IPv6 ICMP Type fields are allocated using an IESG Approval or Standards Action processes. Code Values for existing IPv6 ICMP Type fields are allocated using IESG Approval or Standards Action processes. The policy for assigning Code values for new IPv6 ICMP Types should be defined in the document defining the new Type value.

8. IANA Considerations for fields in the UDP header

The UDP header [UDP] contains the following fields that carry values assigned from IANA-managed name spaces: Source and Destination Port.

Both the Source and Destination Port fields use the same namespace. Values in this namespace are assigned following a specification Required, Expert Review, IESG Approval, IETF Consensus, or Standards Action process. Note that some assignments may involve nondisclosure information.

9. IANA Considerations for fields in the TCP header

The TCP header [TCP] contains the following fields that carry values assigned from IANA-managed name spaces: Source and Destination Port, Reserved Bits, and Option Kind.

9.1 TCP Source and Destination Port fields

Both the Source and Destination Port fields use the same namespace. Values in this namespace are assigned following a specification Required, Expert Review, IESG Approval, IETF Consensus, or Standards Action process. Note that some assignments may involve nondisclosure information.

\subsection{Reserved Bits in TCP Header}

The reserved bits in the TCP header are assigned following a Standards Action process. 


\subsection{TCP Option Kind field}

Values in the Option Kind field are assigned following an IESG Approval or Standards Action process.

10. Security Considerations

Security analyzers such as firewalls and network intrusion detection monitors often rely on unambiguous interpretations of the fields described in this memo. As new values for the fields are assigned, existing security analyzers that do not understand the new values may fail, resulting in either loss of connectivity if the analyzer declines to forward the unrecognized traffic, or loss of security if it does forward the traffic and the new values are used as part of an attack. This vulnerability argues for high visibility (which the Standards Action and IETF Consensus processes ensure) for the assignments whenever possible.

11. References

[ADSCP] Meyer, D., "Administratively Scoped IP Multicast", RFC 2365, July 1998 .

[AN80] Postel, J., "Assigned Numbers", RFC 758, August 1979.

[AN81] Postel, J., "Assigned Numbers", RFC 790, september 1981.

[CONS] Narten, T. and H. Alvestrand, "Guidelines for Writing an IANA Considerations section in RFCs", BCP 26, RFC 2434, October 1998 .

[DIFF] Nichols, K., Blake, S., Baker, F. and D. Black, "Definition of the Differentiated Services Field (DS Field) in the IPv4 and IPv6 Headers", RFC 2474, December 1998.

[ECN] Ramakrishnan, K. and S. Floyd, "A Proposal to add Explicit Congestion Notification (ECN) to IP", RFC 2481, January 1999.

[HC] Jacobson, V., "Compressing TCP/IP headers for low-speed serial links", RFC 1144, February 1990.

[ICMP] Postel, J., "Internet Control Message Protocol", STD 5, RFC 792, September 1981 .

[ICMPV6] Conta, A. and S. Deering, "Internet Control Message Protocol (ICMPv6) for the Internet Protocol Version 6 (IPv6)", RFC 2463, December 1998 . 
[IPHC] Degermark, M., Nordgren, S. and B. Pink, "IP Header Compression", RFC 2507, February 1999.

[MASGN] Hinden, $R$. and S. Deering, "IPv6 Multicast Address Assignments", RFC 2375, July 1998.

[MULT] Deering, S., "Host extensions for IP multicasting", RFC 988, July 1986.

[NDV6] Narten, T., Nordmark, E. and W. Simpson, "Neighbor Discovery for IP Version 6 (IPv6)", RFC 2461, December 1998.

[TCP] Postel, J., "Transmission Control Protocol", STD 7, RFC 793, September 1981 .

[UDP] Postel, J., "User Datagram Protocol", STD 6, RFC 768, August 1980 .

[V4] Postel, J., "Internet Protocol", STD 5, RFC 791, September, 1981 .

[V6] Deering, S. and R. Hinden, "Internet Protocol, Version 6 (IPv6) Specification", RFC 2460, December 1998.

[V6AA] IAB, IESG, "IPv6 Address Allocation Management", RFC 1881, December 1995.

[V6AD] Hinden, $R$. and S. Deering, "IP Version 6 Addressing Architecture", RFC 2373, July 1998. 
12. Authors' Addresses

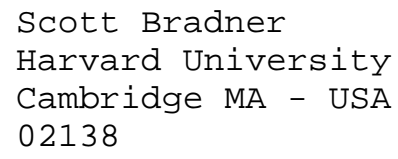


13. Full Copyright statement

Copyright (C) The Internet Society (2000). All Rights Reserved.

This document and translations of it may be copied and furnished to others, and derivative works that comment on or otherwise explain it or assist in its implementation may be prepared, copied, published and distributed, in whole or in part, without restriction of any kind, provided that the above copyright notice and this paragraph are included on all such copies and derivative works. However, this document itself may not be modified in any way, such as by removing the copyright notice or references to the Internet society or other Internet organizations, except as needed for the purpose of developing Internet standards in which case the procedures for copyrights defined in the Internet standards process must be followed, or as required to translate it into languages other than English.

The limited permissions granted above are perpetual and will not be revoked by the Internet society or its successors or assigns.

This document and the information contained herein is provided on an "AS IS" basis and THE INTERNET SOCIETY AND THE INTERNET ENGINEERING TASK FORCE DISCLAIMS ALL WARRANTIES, EXPRESS OR IMPLIED, INCLUDING BUT NOT LIMITED TO ANY WARRANTY THAT THE USE OF THE INFORMATION HEREIN WILL NOT INFRINGE ANY RIGHTS OR ANY IMPLIED WARRANTIES OF MERCHANTABILITY OR FITNESS FOR A PARTICULAR PURPOSE.

Acknowledgement

Funding for the RFC Editor function is currently provided by the Internet Society. 